\title{
A Doublet-Shift Transmitted Reference Scheme for Ultra-Wideband Communication Systems
}

\author{
Muhammad Gufran Khan, Jörgen Nordberg, and Ingvar Claesson \\ Department of Signal Processing, School of Engineering, \\ Blekinge Institute of Technology, SE-372 25 Ronneby, Sweden \\ Email: \{Muhammad.Gufran.Khan, Jorgen.Nordberg, Ingvar.Claesson\} @bth.se
}

\begin{abstract}
The transmitted reference (TR) scheme has gained attention for ultra-wideband (UWB) communications as it bypasses the complex task of channel estimation and provides a simple receiver structure. However, the low-complexity detection in this scheme is achieved at the expense of a loss in performance. To this end, this paper proposes a doublet-shift TR (DsTR) signaling and detection scheme to improve the performance of conventional TR impulse radio UWB system. The performance of the proposed scheme is compared with conventional TR signaling scheme in terms of uncoded bit error rate (BER). The simulation results validate that the doublet-shift TR scheme achieves about $1.5-2 \mathrm{~dB}$ better performance than the conventional TR scheme. The DsTR scheme does not require extra energy and still has the low-complexity.
\end{abstract}

\section{INTRODUCTION}

The UWB technology offers many promising applications, however, UWB research and development has to cope with formidable challenges that limit their bit error rate performance, capacity, throughput, and network flexibility [1]. The impulse radio (IR) UWB utilizes short duration pulses for the transmission and has the ability to resolve individual multipath components [2]. However, the large number of resolvable paths in such a system makes it unrealistic to employ the traditional RAKE receiver to capture a significant portion of the energy contained in the received multipath components [2], [3]. A RAKE receiver that implements tens or even hundreds of correlation operations may be required to take full advantage of the available signal energy [4], [5].

Transmitted reference (TR) scheme proposed by Hoctor and Tomlinson [6] is an alternative simple autocorrelation receiver scheme for the demodulation of IR-UWB signals. In TR systems, the reference signal and data signal are transmitted within the coherence time of the channel, it is assumed that the channel responses to the two signals are the same [7]. TR scheme does not require expensive path-by-path channel estimation as the reference signal is used to demodulate the data signal. In addition, this scheme has the advantage that receiver timing and synchronization requirements are substantially reduced; by transmitting a reference along with the data, it is possible to eliminate the need for locally generated reference (LGR) and the complicated issue of LGR synchronization [8]. The main drawback of the TR scheme stems from a reduced signal-to-noise ratio, which is partly due to "wasting" energy on the reference pulses that are non-information-bearing [9]. The second drawback of these receivers is the significant performance degradation associated with employing noisy received signals as the reference signals for data detection [2].

In [10], an averaged TR (ATR) system which performs averaging of the previously received reference pulses is proposed to decrease the noise in the reference template. The suppression of noise by averaging previously received reference pulses requires the implementation of precise delays which may be burdensome [10]. Alternatively, the averaging process might need to be done using digital signal processing; in this situation, the receiver must have a high-sampling-frequency ADC, and the receiver structure is no longer "simple" [12]. In [7], optimal and suboptimal UWB TR receivers are analyzed and a differential TR system is presented in which no references are transmitted. However, the differential TR system also suffers from the noisy reference template without averaging of the pulses.

Further, some other schemes has been proposed in the literature to reduce the performance loss in the conventional TR UWB system, e.g., in [11], an energy efficient modulation scheme is proposed where the reference pulse also carries information. In [12], a generalized model of TR scheme which combines the conventional TR and differential TR techniques has been presented to increase the power efficiency and improve bit error probability (BEP). A signal processing model for a TR UWB system is proposed in [13], for the case where both pulses in a doublet are more closely spaced than the length of the impulse response. [14] has also presented a dual pulse transmission and auto-correlation detection scheme for UWB communications.

In this paper, a doublet-shift TR (DsTR) signaling and detection scheme is proposed for IR-UWB systems. The proposed scheme transmits two TR doublets and shifts the positions of the pulses in the latter doublet. The DsTR scheme utilizes the same energy as the conventional TR scheme. The structures of the receivers for the DsTR scheme are "simple" and similar to the conventional TR receiver, which means that the delay and correlation can be performed in analog domain. The performance of the proposed scheme is evaluated over the standard IEEE 802.15.4a multipath channels. The performance is compared with conventional TR scheme in terms of uncoded BER. The simulation results validate that the use of a doubletshift TR signaling scheme can improve the performance over conventional TR signaling scheme. 
The rest of the paper is organized as follows. In section II, the signal model of conventional and proposed TR IRUWB systems is presented. Section III describes the receiver structures for the detection of conventional TR and DsTR signaling schemes. The performance evaluation and the results are discussed in section IV and finally the conclusions are presented in section $\mathrm{V}$.

\section{Signal Model}

\section{A. Conventional TR Scheme}

For conventional TR IR-UWB system, the transmitted signal using binary antipodal modulation can be written as

$$
\begin{aligned}
s(t)= & \sqrt{\frac{E_{b}}{2 N_{f}}} \sum_{j=-\infty}^{\infty}\left[p\left(t-j T_{f}-c_{j} T_{c}\right)\right. \\
& \left.+b_{\left\lfloor j / N_{f}\right\rfloor} p\left(t-j T_{f}-c_{j} T_{c}-T_{d}\right)\right],
\end{aligned}
$$

where $p(t)$ is the transmitted Gaussian monocycle, $E_{b}$ is the symbol energy, $T_{c}$ is the chip duration, $T_{f}$ is the frame duration. Fig. 1 shows an example of the transmitted sequence for conventional TR scheme. The figure explains that each transmitted bit contains $N_{f}$ successive frames and each frame contains a TR doublet consisting of two pulses separated by a fixed delay of duration $T_{d}$. Thus, one binary information symbol $b_{\left\lfloor j / N_{f}\right\rfloor} \in\{1,-1\}$ is transmitted by a stream of $2 N_{f}$ pulses. The $c_{j} \in\left\{0,1, \ldots, N_{c}-1\right\}$ is a periodic pseudorandom time-hopping sequence, which is used to time-hop the pairs of reference and data-modulated pulses in each frame.

The signal is transmitted over a multipath channel. The impulse response of a simplified multipath channel can be written as

$$
h(t)=\sum_{l=0}^{L-1} \alpha_{l} \delta\left(t-\tau_{l}\right),
$$

where $\alpha_{l}$ are the channel tap weights, $L$ is the number of multipath components and $\tau_{l}$ is the delay associated with $l$ th multipath component. The received signal can then be expressed as

$$
\begin{aligned}
r(t)= & \sqrt{\frac{E_{b}}{2 N_{f}}} \sum_{j=-\infty}^{\infty} \sum_{l=0}^{L-1}\left[\alpha_{l} p\left(t-j T_{f}-c_{j} T_{c}-\tau_{l}\right)\right. \\
& \left.+b_{\left\lfloor j / N_{f}\right\rfloor} \alpha_{l} p\left(t-j T_{f}-c_{j} T_{c}-T_{d}-\tau_{l}\right)\right]+n(t) \\
= & \sqrt{\frac{E_{b}}{2 N_{f}}} \sum_{j=-\infty}^{\infty}\left[g\left(t-j T_{f}-c_{j} T_{c}\right)\right. \\
& \left.+b_{\left\lfloor j / N_{f}\right\rfloor} g\left(t-j T_{f}-c_{j} T_{c}-T_{d}\right)\right]+n(t)
\end{aligned}
$$

where $g(t)$ is interpreted as the aggregate channel after convolving with the transmitted pulse, $g(t)=p(t) * h(t)$, i.e.,

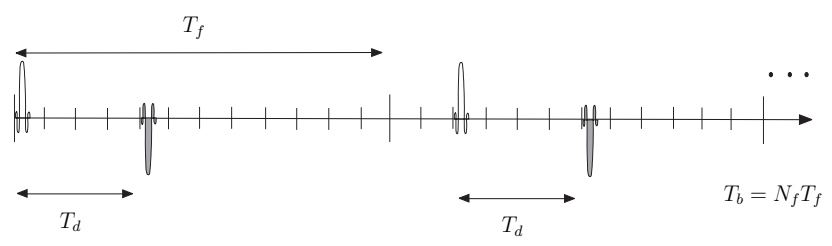

Fig. 1. An example of the transmitted sequence of conventional TR scheme for one bit, where the data pulses are shown filled and $N_{f}=2$ in this figure.

$$
g(t)=\sum_{l=0}^{L-1} \alpha_{l} p\left(t-\tau_{l}\right),
$$

$p(t)$ is the transmitted pulse and $n(t)$ is a Gaussian process with zero mean and variance $\sigma_{n}^{2}$. The duration of $g(t)$ is defined as $T_{g}=T_{p}+T_{m d s}$, where $T_{p}\left(\leq T_{c}\right)$ is the pulse duration, $T_{m d s}$ is the maximum delay spread of the channel. The interference between reference and modulated pulses can be avoided by keeping the delay $T_{g} \leq T_{d}$ and $2 T_{d} \leq T_{f}$.

\section{B. Doublet-shift TR Scheme}

The transmitted signal in the proposed doublet-shift TR signaling scheme for IR-UWB system using binary antipodal modulation can be written as

$$
\begin{aligned}
\bar{s}(t)= & \sqrt{\frac{E_{b}}{4 \bar{N}_{f}}} \sum_{j=-\infty}^{\infty}\left[p\left(t-j \bar{T}_{f}-c_{j} \bar{T}_{c}\right)\right. \\
& +b_{\left\lfloor j / N_{f}\right\rfloor} p\left(t-j \bar{T}_{f}-c_{j} \bar{T}_{c}-\Delta \bar{T}_{d}\right) \\
& +b_{\left\lfloor j / N_{f}\right\rfloor} p\left(t-j \bar{T}_{f}-c_{j} \bar{T}_{c}-\bar{T}_{d}\right) \\
& \left.+p\left(t-j \bar{T}_{f}-c_{j} \bar{T}_{c}-\Delta \bar{T}_{d}-\bar{T}_{d}\right)\right],
\end{aligned}
$$

where, as in (1), $p(t)$ is the transmitted Gaussian monocycle, $E_{b}$ is the symbol energy, $\bar{T}_{c}\left(T_{p} \leq \bar{T}_{c} \leq T_{c}\right)$ is the chip duration, and $\bar{T}_{f}\left(\geq T_{f}\right)$ is the frame duration. Fig. 2 shows the signaling sequence for the DsTR schemes and also illustrates that each transmitted bit contains $\bar{N}_{f}\left(=N_{f} / 2\right)$ successive frames (i.e., half of the conventional TR scheme) and each frame contains four pulses. The four pulses are further divided into two doublets (or TR doublets), with each doublet carrying two pulses. The first doublet transmits the reference pulse first and the data-modulated pulse follows. In the second doublet, the order of the pulses within the doublet is shifted, i.e., the data-modulated pulse is transmitted before the reference pulse. The doublets are separated by a fixed delay of duration $\bar{T}_{d}$ and the pulses within a doublet are separated by a fixed delay $\Delta \bar{T}_{d}$. The DsTR scheme uses periodic pseudo-random time-hopping sequence $c_{j} \in\left\{0,1, \ldots, N_{c}-1\right\}$ to time-hop the pairs of the TR doublets in each frame.

In DsTR scheme, $\bar{T}_{d}$ and $\Delta \bar{T}_{d}$ can be kept according to the channel delay spread. In case of $T_{c}=2 T_{p}$, a doublet can be transmitted within a chip duration by interleaving the pulses in time (i.e., $\Delta \bar{T}_{d}=T_{p}$ ) and allowing the IPI to occur. For convenience of notation in rest of the paper, the DsTR 


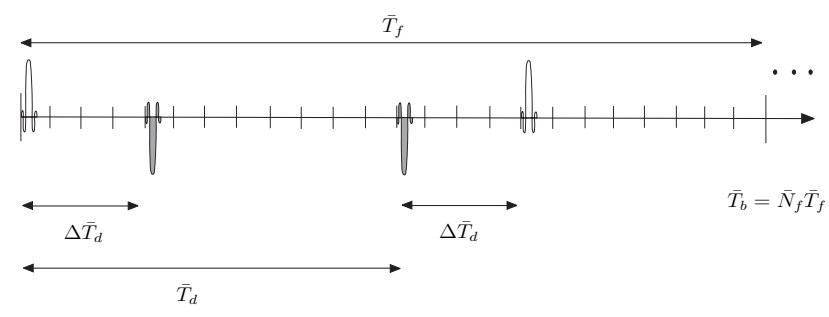

(a)

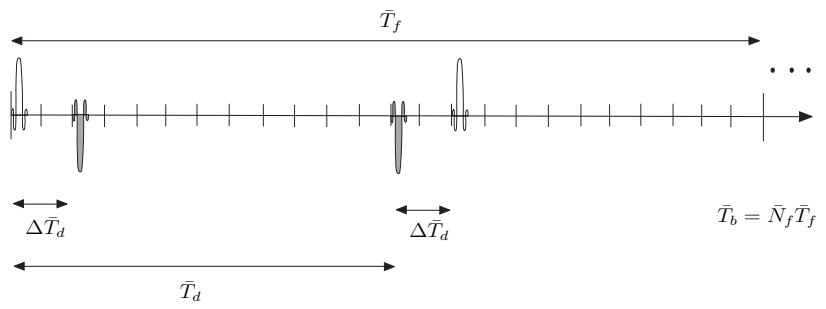

(b)

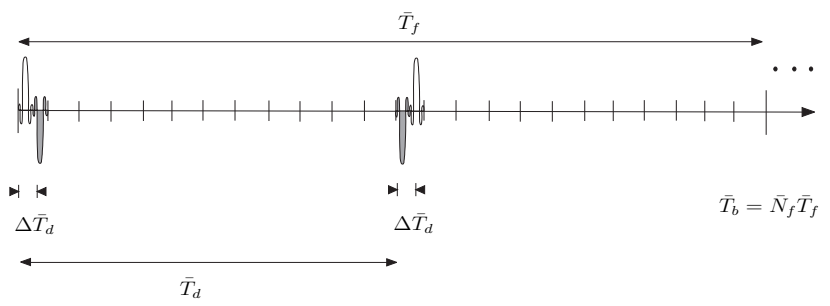

(c)

Fig. 2. An example of the transmitted sequence of doublet-shift schemes for one bit, where the data pulses are shown filled and $\bar{N}_{f}=1$ in figures. (a) For DsTR-(a) scheme, $\Delta \bar{T}_{d} \geq T_{g}$. (b) For DsTR-(b) scheme, $T_{p}<\Delta \bar{T}_{d}<T_{g}$. (c) For DsTR-(c) scheme, $\Delta T_{d}=T_{p}$.

signaling schemes shown in Fig. 2, for the case of $\Delta \bar{T}_{d} \geq T_{g}$, $T_{p}<\Delta \bar{T}_{d}<T_{g}$ and $\Delta \bar{T}_{d}=T_{p}$ are denoted as DsTR-(a), DsTR-(b) and DsTR-(c), respectively. It is evident from the comparison of Fig. 1 and Fig. 2 that the total number of TR doublets utilized per bit is the same for the conventional TR scheme and the DsTR scheme. In other words, the amount of energy assigned per bit is the same in both the cases as both schemes use the same number of Gaussian monocycles per bit, as also illustrated by (5).

In a similar fashion as (3), the received signal after passing through the multipath channel can be written as

$$
\begin{aligned}
\bar{r}(t)= & \sqrt{\frac{E_{b}}{4 \bar{N}_{f}}} \sum_{j=-\infty}^{\infty}\left[g\left(t-j \bar{T}_{f}-c_{j} \bar{T}_{c}\right)\right. \\
& +b_{\left\lfloor j / N_{f}\right\rfloor} g\left(t-j \bar{T}_{f}-c_{j} \bar{T}_{c}-\Delta \bar{T}_{d}\right) \\
& +b_{\left\lfloor j / N_{f}\right\rfloor} g\left(t-j \bar{T}_{f}-c_{j} \bar{T}_{c}-\bar{T}_{d}\right) \\
& \left.+g\left(t-j \bar{T}_{f}-c_{j} \bar{T}_{c}-\Delta \bar{T}_{d}-\bar{T}_{d}\right)\right]+\bar{n}(t) \\
= & \bar{r}_{1}(t)+\bar{r}_{2}(t),
\end{aligned}
$$

where $\bar{r}(t)$ is expressed as a linear combination of two signals $\bar{r}_{1}(t)$ and $\bar{r}_{2}(t)$, such that

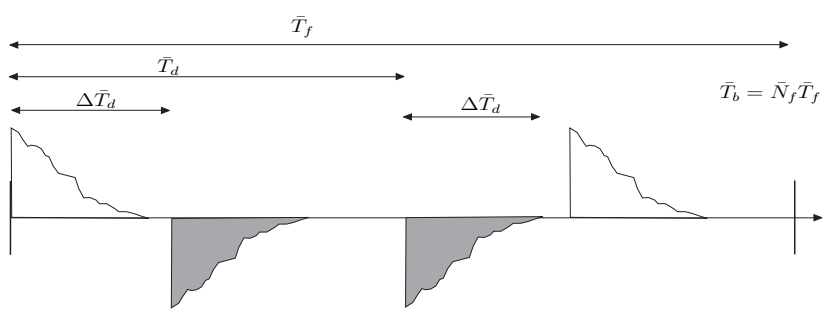

(a)

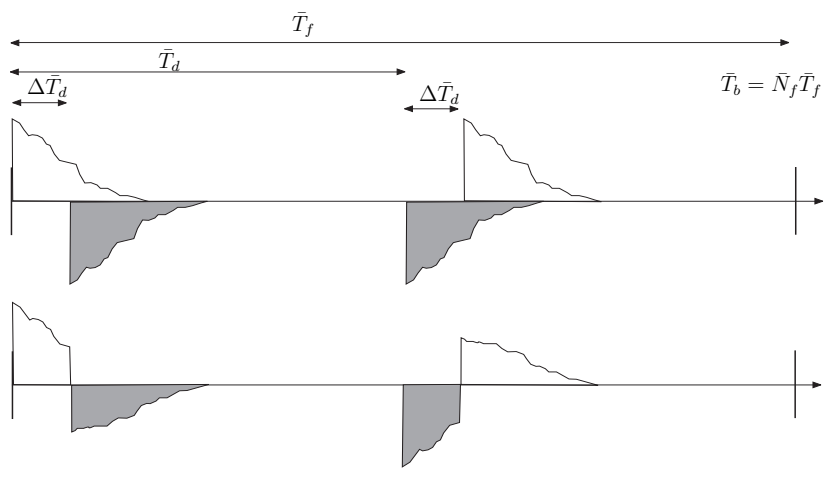

(b)

Fig. 3. An illustration of the DsTR scheme with (a) no IPI and (b) IPI within a doublet, assuming that the channel is static over a bit interval

$$
\begin{aligned}
\bar{r}_{1}(t)= & \sqrt{\frac{E_{b}}{2 \bar{N}_{f}}} \sum_{j=-\infty}^{\infty}\left[g\left(t-j \bar{T}_{f}-c_{j} \bar{T}_{c}\right)\right. \\
& \left.+b_{\left\lfloor j / N_{f}\right\rfloor} g\left(t-j \bar{T}_{f}-c_{j} \bar{T}_{c}-\Delta \bar{T}_{d}\right)\right]+\bar{n}_{1}(t),
\end{aligned}
$$

and

$$
\begin{aligned}
\bar{r}_{2}(t)= & \sqrt{\frac{E_{b}}{2 \bar{N}_{f}}} \sum_{j=-\infty}^{\infty}\left[b_{\left\lfloor j / N_{f}\right\rfloor} g\left(t-j \bar{T}_{f}-c_{j} \bar{T}_{c}-\bar{T}_{d}\right)\right. \\
& \left.+g\left(t-j \bar{T}_{f}-c_{j} \bar{T}_{c}-\Delta \bar{T}_{d}-\bar{T}_{d}\right)\right]+\bar{n}_{2}(t),
\end{aligned}
$$

where $\bar{r}_{1}(t)$ carries the first TR doublets and $\bar{r}_{2}(t)$ consists of the second shifted TR doublets, $\bar{n}_{1}(t)$ and $\bar{n}_{2}(t)$ are Gaussian processes with zero mean and variance $\sigma_{n}^{2}$. In this case, the interference between any two doublets can be avoided by keeping the delay $\left(\Delta \bar{T}_{d}+T_{g}\right) \leq \bar{T}_{d}$ and $2 \bar{T}_{d} \leq \bar{T}_{f}$. Fig. 3 illustrates the DsTR scheme after passing through the multipath channel, assuming that the channel is static over a bit interval.

\section{RECEIVER STRUCTURES}

The received signal is passed through a filter which is matched to the transmitted pulse. For simplicity, it is assumed that the shape of the received pulse is the same as the 


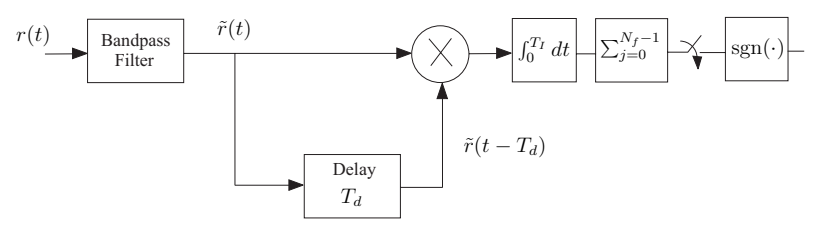

Fig. 4. Structure of the conventional TR receiver.

transmitted pulse. The output of the filter for conventional TR scheme, denoted by $\tilde{r}(t)$, is given as

$$
\begin{aligned}
\tilde{r}(t)= & \sqrt{\frac{E_{b}}{2 N_{f}}} \sum_{j=-\infty}^{\infty}\left[\tilde{g}\left(t-j T_{f}-c_{j} T_{c}\right)\right. \\
& \left.+b_{\left\lfloor j / N_{f}\right\rfloor} \tilde{g}\left(t-j T_{f}-c_{j} T_{c}-T_{d}\right)\right]+\tilde{n}(t),
\end{aligned}
$$

where $\tilde{g}(t)$ and $\tilde{n}(t)$ are filtered versions of $g(t)$ and $n(t)$, respectively. In a similar manner, the output of the filter for the DsTR scheme can be written as

$$
\tilde{\bar{r}}(t)=\tilde{\bar{r}}_{1}(t)+\tilde{\bar{r}}_{2}(t) .
$$

\section{A. Conventional TR Receiver}

The conventional TR receiver delays the signal $\tilde{r}(t)$ and correlates it with itself, as shown in Fig. 4. The outputs of the correlator are summed over the $N_{f}$ frames to acquire the decision statistic. Assuming ideal synchronization, the decision statistic for TR signaling [15] is given by

$$
Z_{T R}=\sum_{j=0}^{N_{f}-1} \int_{j T_{f}+c_{j} T_{c}+T_{d}}^{j T_{f}+c_{j} T_{c}+T_{d}+T_{I}} \tilde{r}(t) \tilde{r}\left(t-T_{d}\right) d t
$$

where $T_{I}$ is the integration interval in each frame which satisfies $0<T_{I} \leq T_{g}$. The bit decision is made using conventional detection as $\hat{b}=\operatorname{sgn}\left(Z_{T R}\right)$, where $\operatorname{sgn}(\cdot)$ stands for the sign function.

\section{B. Doublet-shift TR Receiver}

For the detection of DsTR signals, the structure of the first receiver denoted as DsTR-I is the same as the conventional TR receiver, see Fig. 5(a). In DsTR-I receiver, the first TR doublet is used as a reference template for the second shifted TR doublet. First, the signal $\tilde{\bar{r}}(t)$ is delayed by $\bar{T}_{d}$, which is the delay between two subsequent TR doublets and then the correlation is performed. The decision statistic for DsTR signaling is formed as

$$
Z_{D s T R}=\sum_{j=0}^{\bar{N}_{f}-1} \int_{j \bar{T}_{f}+c_{j} \bar{T}_{c}+\bar{T}_{d}}^{j \bar{T}_{f}+c_{j} \bar{T}_{c}+\bar{T}_{d}+\bar{T}_{I}} \tilde{\bar{r}}(t) \tilde{\bar{r}}\left(t-\bar{T}_{d}\right) d t
$$

As $\bar{r}(t)$ can be expressed as a linear combination of two signals $\bar{r}_{1}(t)$ and $\bar{r}_{2}(t)$, then

$$
\begin{aligned}
Z_{D s T R}= & \sum_{j=0}^{\bar{N}_{f}-1} \int_{j \bar{T}_{f}+c_{j} \bar{T}_{c}+\bar{T}_{d}}^{j \bar{T}_{f}+c_{j} \bar{T}_{c}+\bar{T}_{d}+\bar{T}_{I}}\left(\tilde{\bar{r}}_{1}(t)+\tilde{\bar{r}}_{2}(t)\right) \\
& \cdot\left(\tilde{\bar{r}}_{1}\left(t-\bar{T}_{d}\right)+\tilde{\bar{r}}_{2}\left(t-\bar{T}_{d}\right)\right) d t \\
= & \sum_{j=0}^{\bar{N}_{f}-1} \int_{j \bar{T}_{f}+c_{j} \bar{T}_{c}+\bar{T}_{d}}^{j \bar{T}_{f}+c_{j} \bar{T}_{c}+\bar{T}_{d}+\bar{T}_{I}}\left[\tilde{\bar{r}}_{1}(t) \tilde{\bar{r}}_{1}\left(t-\bar{T}_{d}\right)\right. \\
& +\tilde{\bar{r}}_{1}(t) \tilde{\bar{r}}_{2}\left(t-\bar{T}_{d}\right)+\tilde{\bar{r}}_{1}\left(t-\bar{T}_{d}\right) \tilde{\bar{r}}_{2}(t) \\
& \left.+\tilde{\bar{r}}_{2}(t) \tilde{\bar{r}}_{2}\left(t-\bar{T}_{d}\right)\right] d t,
\end{aligned}
$$

where $\bar{T}_{I}$ is the integration interval in each frame which satisfies $0<\bar{T}_{I} \leq\left(\Delta \bar{T}_{d}+T_{g}\right)$. The output of multiplier of the correlator consists of signal regions and noise-only regions, as illustrated by (13). However, the integration is performed only overs the signal regions. If $\bar{T}_{d} \geq\left(\Delta \bar{T}_{d}+T_{g}\right)$ and $2 \bar{T}_{d} \leq \bar{T}_{f}$, (13) reduces to a simplified form as

$$
Z_{D s T R}=\sum_{j=0}^{\bar{N}_{f}-1} \int_{j \bar{T}_{f}+c_{j} \bar{T}_{c}+\bar{T}_{d}}^{j \bar{T}_{f}+c_{j} \bar{T}_{c}+\bar{T}_{d}+\bar{T}_{I}} \tilde{\bar{r}}_{2}(t) \tilde{\bar{r}}_{1}\left(t-\bar{T}_{d}\right) d t,
$$

which illustrates that the signal $\tilde{\bar{r}}_{1}(t)$ consisting of the first TR doublets is delayed and used as a reference signal for $\tilde{\bar{r}}_{2}(t)$ that carries the second shifted TR doublets. As Fig. 3(b) shows, the first doublet can serve as a reference template for the second doublet in case of DsTR-(b) and DsTR-(c) signaling schemes also. As the correlation is performed only over the signal region, the use of closely spaced pulses within a doublet improves the SNR over the correlation interval. Thus, the use of DsTR-(b) and DsTR-(c) signaling with DsTR-I structure is expected to lead to performance improvement. The bit decision for DsTR-I structure is made using conventional detection as $\hat{b}_{D s T R-I}=\operatorname{sgn}\left(Z_{D s T R}\right)$.

Further, Fig. 5(b) shows the second receiver structure denoted as DsTR-II. The DsTR-II structure is obtained observing that each TR doublet in DsTR scheme also contains a reference and a corresponding data-modulated pulse, a second correlator can be used in the receiver to demodulate the data-modulated pulses within a doublet. In this case, the use of second correlator is expected to improve the performance as the detection is performed by combining the outputs of both the correlators. Fig. 5(b) shows that the received signal is delayed by $\Delta \bar{T}_{d}$ before the second correlator. The integration in the second correlator is performed over $2 \bar{N}_{f}$ intervals within a bit duration, as the number of doublets is twice the number of frames. In DsTR-II structure, the decision statistic for the second correlator is denoted as $\Delta Z_{D s T R}$ and is computed as

$$
\begin{aligned}
\Delta Z_{D s T R}= & \sum_{j=0}^{\bar{N}_{f}-1}\left[\int_{j \bar{T}_{f}+c_{j} \bar{T}_{c}+\Delta \bar{T}_{d}}^{j \bar{T}_{f}+c_{j} \bar{T}_{c}+\Delta \bar{T}_{d}+\Delta \bar{T}_{I}} \tilde{\bar{r}}(t) \tilde{\bar{r}}\left(t-\Delta \bar{T}_{d}\right)\right. \\
& \left.+\int_{j \bar{T}_{f}+c_{j} \bar{T}_{c}+\Delta \bar{T}_{d}+\bar{T}_{d}}^{j \bar{T}_{f}+c_{j} \bar{T}_{c}+\Delta \bar{T}_{d}+\bar{T}_{d}+\Delta \bar{T}_{I}} \tilde{\bar{r}}(t) \tilde{\bar{r}}\left(t-\Delta \bar{T}_{d}\right)\right] d t
\end{aligned}
$$


Again, as $\bar{r}(t)$ can be written as a linear combination of $\bar{r}_{1}(t)$ and $\bar{r}_{2}(t)$, then

$$
\begin{aligned}
\Delta Z_{D s T R}= & \sum_{j=0}^{\bar{N}_{f}-1}\left[\int_{j \bar{T}_{f}+c_{j} \bar{T}_{c}+\Delta \bar{T}_{d}}^{j \bar{T}_{f}+c_{j} \bar{T}_{c}+\Delta \bar{T}_{d}+\Delta \bar{T}_{I}}\left(\tilde{\bar{r}}_{1}(t)+\tilde{\bar{r}}_{2}(t)\right)\right. \\
& \cdot\left(\tilde{\bar{r}}_{1}\left(t-\Delta \bar{T}_{d}\right)+\tilde{\bar{r}}_{2}\left(t-\Delta \bar{T}_{d}\right)\right) d t \\
& +\int_{j \bar{T}_{f}+c_{j} \bar{T}_{c}+\Delta \bar{T}_{d}+\bar{T}_{d}}^{j \bar{T}_{f}+c_{j} \bar{T}_{c}+\Delta \bar{T}_{d}+\bar{T}_{d}+\Delta \bar{T}_{I}}\left(\tilde{\bar{r}}_{1}(t)+\tilde{\bar{r}}_{2}(t)\right) \\
& \left.\cdot\left(\tilde{\bar{r}}_{1}\left(t-\Delta \bar{T}_{d}\right)+\tilde{\bar{r}}_{2}\left(t-\Delta \bar{T}_{d}\right)\right)\right] d t
\end{aligned}
$$

where $\Delta \bar{T}_{I}$ is the integration interval within each doublet which satisfies $0<\Delta \bar{T}_{I} \leq T_{g}$. If $\bar{T}_{d} \geq\left(\Delta \bar{T}_{d}+T_{g}\right)$ and $2 \bar{T}_{d} \leq \bar{T}_{f}$, (16) reduces to a simplified form as

$$
\begin{aligned}
& \Delta Z_{D s T R} \\
& \quad=\sum_{j=0}^{\bar{N}_{f}-1}\left[\int_{j \bar{T}_{f}+c_{j} \bar{T}_{c}+\Delta \bar{T}_{d}}^{j \bar{T}_{f}+c_{j} \bar{T}_{c}+\Delta \bar{T}_{d}+\Delta \bar{T}_{I}}\left(\tilde{\bar{r}}_{1}(t) \tilde{\bar{r}}_{1}\left(t-\Delta \bar{T}_{d}\right)\right) d t\right. \\
& \left.\quad+\int_{j \bar{T}_{f}+c_{j} \bar{T}_{c}+\Delta \bar{T}_{d}+\bar{T}_{d}}^{j \bar{T}_{f}+c_{j} \bar{T}_{c}+\Delta \bar{T}_{d}+\bar{T}_{d}+\Delta \bar{T}_{I}}\left(\tilde{\bar{r}}_{2}(t) \tilde{\bar{r}}_{2}\left(t-\Delta \bar{T}_{d}\right)\right)\right] d t
\end{aligned}
$$

where the first term illustrates that the reference pulses in the first doublets are delayed and correlated with their corresponding data-modulated pulses. Similarly, the second term shows that, in the second shifted doublets, the data-modulated pulses are delayed and correlated with their corresponding reference pulses. For DsTR-II structure, as shown in Fig. 5(b), the decision statistics from the two correlators are added and the bit decision is made as $\hat{b}_{D s T R-I I}=\operatorname{sgn}\left(Z_{D s T R}+\Delta Z_{D s T R}\right)$

\section{Performance Evaluation}

The system is simulated on the multipath channels proposed by IEEE 802.15.4a [16] for low-data-rate UWB systems. The channel model CM1 is used which covers residential lineof-sight (LOS) environments with maximum delay spread of about $100 \mathrm{~ns}$. The binary antipodal modulation is used employing second derivative of the Gaussian pulse. The pulse duration is kept about $1.4 \mathrm{~ns}$. The uncoded data rate of $0.5 \mathrm{Mbps}$ is achieved with $N_{f}=10$ and $T_{f}=200 \mathrm{~ns}$ for TR scheme, and $\bar{N}_{f}=5$ and $\bar{T}_{f}=400 \mathrm{~ns}$ for DsTR schemes. For TR scheme, $T_{d}=100 \mathrm{~ns}$; for DsTR schemes, $\bar{T}_{d}=200 \mathrm{~ns}$ and $\Delta \bar{T}_{d}$ is varied according to the signaling scheme used $\left(\bar{T}_{d}\right.$ and $\Delta \bar{T}_{d}$ can be selected according to the channel delay spread). The energy of the channel impulse response is normalized as $\sum \alpha_{l}^{2}=1$ and the system is supposed to be perfectly synchronized.

The simulated uncoded BER curves are shown in Fig. 6 for TR receiver which is using conventional TR signaling and the DsTR-I and DsTR-II receiver structures using DsTR-(a) signaling scheme with $\Delta \bar{T}_{d}=100 \mathrm{~ns}$. The integration intervals $\bar{T}_{I}=50 \mathrm{~ns}$ and $\Delta \bar{T}_{I}=50 \mathrm{~ns}$. The results show that the DsTR-I structure has almost the same BER performance

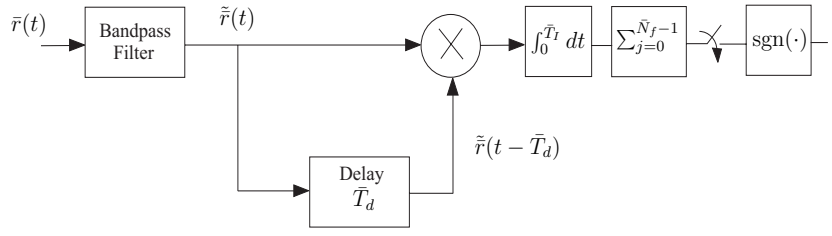

(a)

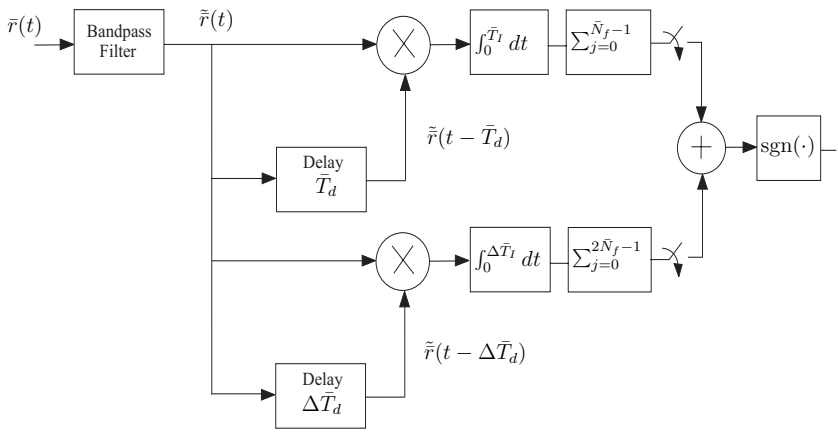

(b)

Fig. 5. (a) Structure of the DsTR-I receiver, (b) structure of the DsTR-II receiver.

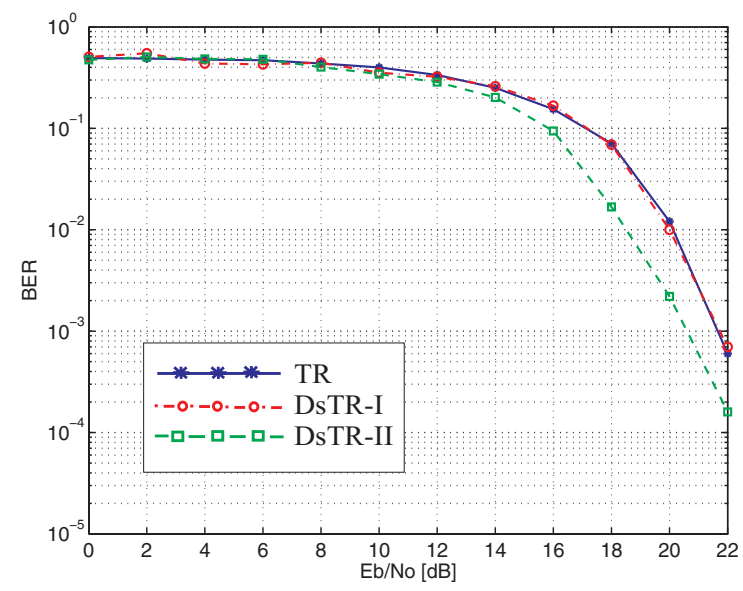

Fig. 6. Uncoded BER curves of conventional TR, and DsTR-I and DsTR-II receivers for DsTR-(a) signaling scheme.

as the conventional TR receiver. However, DsTR-II receiver outperforms both TR and DsTR-I receiver structures by about $1-1.5 \mathrm{~dB}$. It is intuitive that TR and DsTR-I exhibit the same performance as both receivers not only have the same structure but also both demodulate almost similar signaling sequences ( only positions of the pulses are shifted in the latter doublet in DsTR-(a)). It can be concluded that DsTR-II structure provides gain in combination with DsTR-(a) signaling scheme.

In order to evaluate the performance improvement using the DsTR-(b) signaling scheme, the simulation has been performed with $\Delta \bar{T}_{d}=25 \mathrm{~ns}$, which means that the pulses within a doublet are spaced less than the maximum delay spread of the channel. The integration intervals $\bar{T}_{I}=50 \mathrm{~ns}$ and $\Delta \bar{T}_{I}=25$ ns. The results in Fig. 7 depict that both DsTR- 


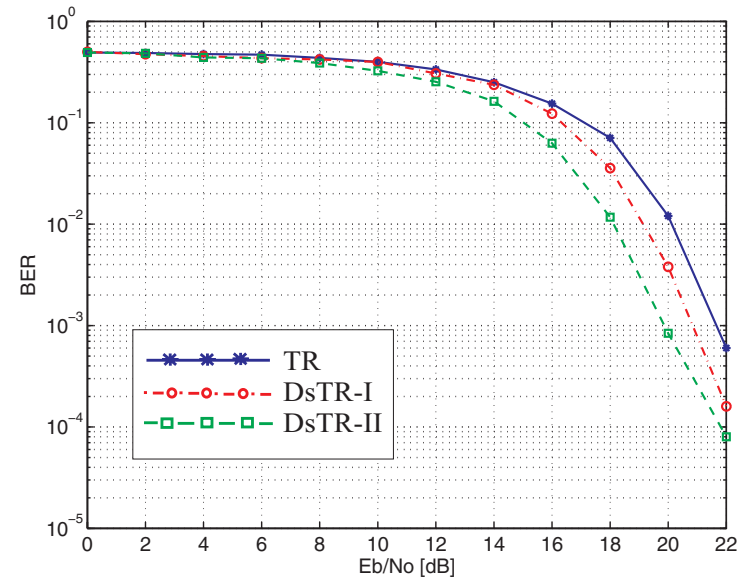

Fig. 7. Uncoded BER curves of conventional TR, and DsTR-I and DsTR-II receivers for DsTR-(b) signaling scheme.

I and DsTR-II structures show improvement in performance for DsTR-(b) scheme. The curves indicate that DsTR-I and DsTR-II receivers outperform TR receiver by about $1 \mathrm{~dB}$ and $2 \mathrm{~dB}$, respectively, which means that the use of closely spaced pulses within a doublet has lead to performance improvement.

Fig. 8 depicts the uncoded BER curves for the DsTR-I and DsTR-II receiver structures using DsTR-(c) signaling scheme with two pulses within a doublet interleaved in time i.e., $\Delta \bar{T}_{d}=T_{p}=1.4 \mathrm{~ns}$, and $\bar{T}_{I}=50 \mathrm{~ns}$ and $\Delta \bar{T}_{I}=8 \mathrm{~ns}$. It is noteworthy that the DsTR-I structure outperforms by about $1.5 \mathrm{~dB}$ than the conventional TR receiver in this particular case. The results validate that the DsTR-II structure improves the performance by about $2 \mathrm{~dB}$. These results are also quite intuitive as the pulses within a doublet are interleaved in time in DsTR-(c) scheme, DsTR-II structure provides relatively less advantage in this scheme due to IPI within a doublet. Finally, the BER comparison between signaling schemes shows that the DsTR-(c) signaling scheme exhibits better performance among the DsTR schemes for both DsTR-I and DsTR-II structures.

\section{CONCLUSIONS}

A doublet-shift TR signaling and detection scheme is presented for IR-UWB systems. The proposed scheme transmits two TR doublets within a frame duration and shifts the positions of the pulses in the latter doublet. The amount of energy utilized by the proposed scheme is the same as the conventional TR scheme. The receiver structure DsTR-I for the proposed scheme is the same as the conventional TR receiver. The improved DsTR-II structure still has low-complexity as it requires only an extra delay and correlation operation. The simulation results validate that the use of doublet-shift TR signaling scheme can improve the uncoded BER performance by about $1.5-2 \mathrm{~dB}$ over conventional TR signaling scheme with a slight increase in complexity.

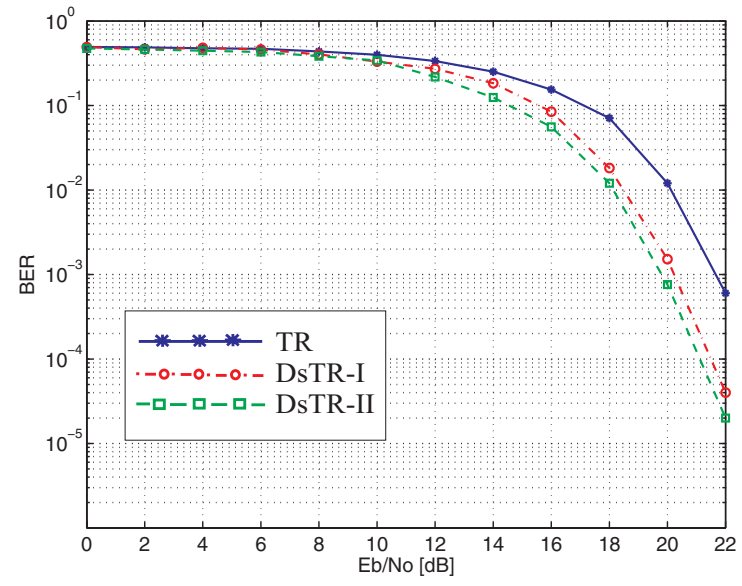

Fig. 8. Uncoded BER curves of conventional TR, and DsTR-I and DsTR-II receivers for DsTR-(c) signaling scheme.

\section{REFERENCES}

[1] L. Yang and G. B. Giannakis, "Ultra-wideband communications," IEEE Signal Process. Magazine, vol. 21, no. 6, pp. 2654, Nov. 2004.

[2] R. C. Qiu, H. Liu, and X. Shen, "Ultra-wideband for multiple access communications," IEEE Comm. Magazine, vol. 43, no. 2, pp. 8087, Feb. 2005.

[3] M. G. Khan, J. Nordberg, A. Mohammed, and I. Claesson, "Performance evaluation of RAKE receiver for UWB systems using measured channels in industrial environments," AusWireless'06, March 2006.

[4] M. Z. Win and R. A. Scholtz, "On the energy capture of ultra-wide bandwidth signals in dense multipath environments," IEEE Commun. Lett., vol. 2, Sep. 1998, pp. 245-247.

[5] M. G. Khan, A. A. Ashraf, J. Karedal, F. Tufvesson, and A. F. Molisch, "Measurements and Analysis of UWB Channels in Industrial Environments," in Proc. WPMC'05, Aalborg, Denmark, Sept. 2005.

[6] R. Hoctor and H. Tomlinson, "Delay-hopped transmitted-reference RF communications," IEEE UWBST, pp. 265269, 2002, Baltimore, MD.

[7] Y.-L. Chao and R. A. Scholtz "Optimal and suboptimal receivers for ultra-wideband transmitted reference systems,'IEEE Global Telecommun. Conf.'03, vol. 2, pp. 759-763, 1-5 Dec. 2003.

[8] W. M. Gifford and M. Z. Win, "On transmitted-reference UWB communications,"Asilomar Conference on Signals, Systems and Computers, 2004., vol. 2, pp. 1526-1531, Nov. 2004.

[9] F. Tufvesson and A. F. Molisch, "Ultra-wideband communication using hybrid matched filter correlation receivers," Proc. IEEE Veh. Technol. Conf., vol. 3, pp. 1290- 1294, May 2004.

[10] D. J. Choi and W. E. Stark, "Performance of ultra-wideband communications with suboptimal receivers in multipath channels,"IEEE Journal of Sleceted Areas Comm., vol. 20, no. 9, Dec. 2002, 1754-1766.

[11] T. Zasowski, F. Althaus, and A. Wittneben, "An energy efficient transmitted-reference scheme for ultra wideband communications,"in Proc. of 2004 UWBST., Kyoto, Japan, May 2004.

[12] Y. -L. Chao and R. A. Scholtz "Novel UWB transmitted reference schemes,"Asilomar Conference on Signals, Systems and Computers, 2004., vol. 1, pp. 652-656, 7-10 Nov. 2004.

[13] Q. H. Dang, A. Trindade, and A.-J. van der Veen, "Signal Model and Receiver Algorithms for a Transmit-Reference Ultra-Wideband Communication System,'IEEE Journal of Sleceted Areas Comm., vol. 24, no. 4, April 2006, 773-779.

[14] X. Dong, A. C. Y. Lee, and L. Xiao, "A new UWB dual pulse transmission and detection technique,"in Proc. IEEE ICC'05., May 2005.

[15] T. Q. S. Quek, M. Z. Win, and D. Dardari, "UWB transmitted reference signaling schemes - part I: performance analysis," Proc. IEEE Conference ICU 2005., pp. 587- 592, Sep. 2005.

[16] A. F. Molisch et al., "IEEE 802.15.4a channel model - final report," Tech. Rep. Document IEEE 802.15-04-0662-02-004a, 2005. 